Article original

\title{
Performance de deux techniques d'extraction des phénols racinaires pour l'évaluation du marquage de la tolérance à la fusariose des clones de palmier a huile (Elaeis guineensis Jacq.)
}

\author{
Sekou Diabate ${ }^{*}$, ${ }^{*}$ Kouakou E. Konan ${ }^{2}$, Désiré Allou ${ }^{1}$, Ouolo A. Coulibaly ${ }^{3}$ \& Hubert De FranqueVILLE ${ }^{4}$ \\ ${ }^{1}$ Centre National de recherche Agronomique (CNRA), Laboratoire Central de Biotechnologie 01 B.P.1740 Abidjan 01 \\ ${ }^{2}$ Centre National de recherche Agronomique (CNRA), Station de Recherche de La Mé 13 B.P.989 Abidjan 13; COTE D'IVOIRE \\ ${ }^{3}$ Centre National de recherche Agronomique (CNRA), Station d'expérimentation et de Production B.P. 8 Dabou; COTE D'IVOIRE \\ ${ }^{4}$ CIRAD, TA 80/02, Avenue Agropolis F34398 Monpellier; CEDEX 5 - FRANCE \\ *Auteur pour les correspondances (E-mail: sekou_diabate2002@yahoo.fr) \\ Reçu le 21-02-2008, accepté le 10-03-2009.
}

\begin{abstract}
Résumé
Les premières études sur les mécanismes de défense du palmier à huile ont mis en évidence le rôle des composés phénoliques dans la tolérance à la fusariose des clones et croisements à partir des racines inoculées par l'agent pathogène. Notre travail a consisté d'une part à améliorer les premières techniques d'extraction en remplaçant l'Ethanol par un mélange Ethanol-eau et d'autre part à améliorer les techniques de microanalyses des marqueurs de tolérance à la fusariose. Les résultats ont montré que les modifications apportées ont permis d'accéder à une quantité plus importante de phénols que la méthode classique. En outre, les analyses des phénols totaux et la fongitoxicité ont permis de discriminer les clones tolérants des clones sensibles. Ces nouvelles méthodes nous ont permis d'évaluer plus rapidement la tolérance à la fusariose des clones après inoculation de l'agent pathogène.
\end{abstract}

Mots clés : Palmier à huile - Fusarium - Phénols - Clones - extraction.

\begin{abstract}
Performance of two techniques of extration of root's phenols for the testing of the tolerance marking in the wilt disease of oil palm clones (Elaeis Guineensis Jacq)

The early studies on the defence mechanisms of the oil palm tree put in evidence the role of phenolic compounds in the tolerance of wilt disease of the clones and crossings from the roots inoculated by the pathogenic agent. Our work consists on one hand in improving the first techniques of extraction by replacing the Ethanol by a mixing Ethanol-water and on the other hand by improving the techniques of microanalyses of the tolerance markers to wilt disease. The results showed that these modifications permitted to reach a quantity more important of phenol than the classic method. Besides, the analyses of the total phenol and the fongitoxicity permitted to discriminate the tolerant clones from the sensitive clones. These new methods allow us to evaluate faster the tolerance to wilt disease of the clones after inoculation of the pathogenic agent.
\end{abstract}

Key-Words: Oil palm tree - Fusarium - Phenols - Clone - extraction. 


\section{Introduction}

Parmi les nombreuses maladies qui affectent la culture du palmier à huile, la fusariose est celle qui revêt le plus d'importance en Afrique tropicale. La Côte d'Ivoire, le Ghana, le Bénin, le Nigeria, le Cameroun, le Congo et le Congo démocratique sont les pays les plus touchés (Franqueville \& Diabaté, 2004). L'agent pathogène de cette trachéomycose

est un champignon tellurique spécifique du palmier à huile : Fusarium oxysporum f. sp. elaeidis (F.O.E.). Les méthodes de lutte sont préventives et se situent essentiellement au niveau du choix du matériel végétal après un test d'inoculation de l'agent pathogène au stade prépépinière (Allou et al., 2003). Tout matériel végétal testé qui a un indice inférieur à 100 est jugé tolérant. Les recherches sur les mécanismes physiologiques de la résistance à la fusariose ont été entreprises pour compléter et apporter une base plus fondamentale au test de prépépinière (Taquet, 1985 ; Diabaté, 1985). Le rôle des composés phénoliques a été mis en évidence dans les racines des plantules inoculées par le F.O.E. après blessures (Diabaté et al., 1992). Cependant, il était difficile de doser avec précision, la totalité de la teneur en phénols des racines des plantules. Pour apporter une solution à ces problèmes qui limitaient ces premières avancées sur les mécanismes de défense du palmier à huile à la fusariose, une nouvelle technique d'extraction des composés phénoliques impliqués dans la réaction de défense du palmier face au fusarium est proposée en comparaison avec l'extraction classique. Les résultats attendus de ces travaux devraient permettre de savoir avec précision la manière de doser les phénols produits lors de la réaction de défense du palmier à huile à la fusariose vasculaire.

\section{Matériel et méthodes}

\subsection{Matériel végétal}

Le matériel végétal étudié est composé de racines de plantules de 6 génotypes issues de la multiplication végétative in vitro appelées clones d'indice fusariose connu. Les clones sont regroupés en trois couples de deux génotypes comportant chacun un génotype sensible et un génotype tolérant (Tableau 1).
Tableau 1: Liste des couples de clones étudiés.

\begin{tabular}{cccc}
\hline Couple & \multicolumn{2}{c}{ Clone } & Im \\
\hline \multirow{2}{*}{ I } & LMC & 022 & 28 \\
& LMC & 074 & 187 \\
II & LMC & 119 & 10 \\
& LMC & 165 & 141 \\
III & LMC & 062 & 42 \\
& LMC & 204 & 163
\end{tabular}

Im = Indice fusariose; Im<100: clone tolérant; Im>100: clone sensible

\subsection{Méthodes d'inoculation au F.O.E et prélèvement d'échantillons racinaires}

Pour l'évaluation de la fongitoxicité des racines, des inoculations de plantules ont été réalisées. La méthode d'inoculation retenue est celle du trempage des racines dans l'inoculum liquide du F.O.E. Cinq essais d'inoculation ont été réalisés uniquement avec le couple LM022/LM074. Dans chaque essai, 5 plantules par clone ont été déterrées pour provoquer des blessures au niveau des racines. Le système racinaire a été ensuite nettoyé puis trempé dans l'inoculum du F.O.E à raison de $10^{7}$ spores par plantule. Les plantules ont été ensuite repiquées dans le terreau. Pour chaque clone, des nombres égaux de plantules non inoculées ont été prélevés et ont constitué les témoins. Cinq délais de prélèvement de racines à analyser ont été retenus (T0, T+1jour, T+3jours, $T+5$ jours et $T+8$ jours après inoculation). Pour chaque délai de prélèvement, les racines des plantules ont été découpées, pesées et conservées au congélateur à $4^{\circ} \mathrm{C}$ pendant 48 heures avant l'extraction des composés phénoliques.

\subsection{Méthodes d'extraction des composés phénoliques}

Deux méthodes d'extraction des phénols ont été utilisées ; une méthode d'extraction classique et une méthode d'extraction nouvelle. Dans la méthode d'extraction classique, $5 \mathrm{~g}$ de racines fraiches ont été découpés et broyés dans $40 \mathrm{ml}$ d'Ethanol pur à l'aide d'un Mixer (Taquet, 1985). La macération des tissus dans l'Ethanol pur a été effectuée à température ambiante pendant 24 heures. Le mélange racines broyées -Ethanol est ensuite filtré. Le filtrat est séché par un Evaporateur rotatif à $40^{\circ} \mathrm{C}$ et repris dans $4 \mathrm{ml}$ d'Ethanol pur. L'extrait ainsi obtenu est conservé au réfrigérateur à $4^{\circ} \mathrm{C}$ avant les dosages. Dans la méthode 
d'extraction nouvelle, $5 \mathrm{~g}$ de racines sont broyés dans l'Azote liquide $\left(-160^{\circ} \mathrm{C}\right)$ et repris dans $40 \mathrm{ml}$ d'un mélange Ethanol-Eau (70-30; $/ \mathrm{v}$ ) en présence de $1 \mathrm{ml}$ d'un antioxydant, le métabisulfite de Sodium (MBS) à $2 \%$ dans l'eau. Ce mélange est porté à ébullition sous vide pendant $30 \mathrm{mn}$. Après refroidissement, le liquide obtenu est filtré et séché par un évaporateur rotatif à $40^{\circ} \mathrm{C}$. Le résidu est repris dans $4 \mathrm{ml}$ du mélange Ethanol-Eau (70-30) $+0,1 \mathrm{ml}$ de MBS à $2 \%$ dans l'eau. L'extrait obtenu est conservé au réfrigérateur à $4^{\circ} \mathrm{C}$ avant les dosages.

\subsection{Quantification des composés phénoliques}

La quantité de phénols contenue dans les extraits racinaires est mesurée par la méthode de Folin ciocalteu (Marigo, 1973). Cette méthode mesure le potentiel d'oxydoréduction des phénols. Les teneurs sont calculées en prenant l'acide chlorogénique comme référence et sont exprimées en mg d'équivalent d'acide chlorogénique par gramme de poids frais.

\subsection{Evaluation de la fongitoxicité des extraits racinaires.}

Les propriétés fongitoxiques des extraits ont été évaluées en milieu liquide, en présence des spores de Fusarium oxysporum f sp. elaeidis (Taquet, 1985). Les tests sont réalisés en flacon d'Erlen meyer $(25 \mathrm{ml})$ régulièrement agité. L'incubation nécessaire à la germination des spores a une durée de 15 heures, à la température de $27^{\circ} \mathrm{C}$. Le résultat des tests est établi sur un total moyen de 150 à 200 spores. Les résultats ont été exprimés en pourcentage d'inhibition calculé par rapport au taux de croissance du témoin sans extrait racinaire.
Taux d'inibition $=\frac{\% \text { de germination du témoin }-\% \text { de germination du motif }}{\% \text { de germination du Témoin }}$

\subsection{Analyses statistiques}

Les analyses statistiques de l'ensemble des données sont effectuées avec les logiciels «ANOVA , XLSTA7.1 » (ADDINSOFT, 2004). Ces logiciels ont permis de faire l'analyse de variance au seuil de $5 \%$ et lorsqu'une différence significative a été mise en évidence, les groupes homogènes ont été constitués à partir du test de NEWMAN-KEULS.

\section{Résultats}

\subsection{Comparaison de la performance des techniques d'extraction pour le marquage de la tolérance des clones}

Les résultats d'analyses du dosage des composés phénoliques racinaires des clones ont montré que la quantité de phénols extraits était significativement plus importante avec la technique d'extraction nouvelle que la technique d'extraction classique. Ces résultats sont confirmés aussi bien avec les clones non inoculés (Tableau 2) qu'avec les clones inoculés (Tableau 3 ). Les résultats d'analyses de l'évolution des phénols après l'inoculation de l'agent pathogène ont aussi révélé que l'extraction par la nouvelle méthode a libéré significativement plus de phénols après dosage que la méthode classique quelque soit le temps d'incubation du Fusarium oxysporum f. sp. elaeidis (Fig. 1).

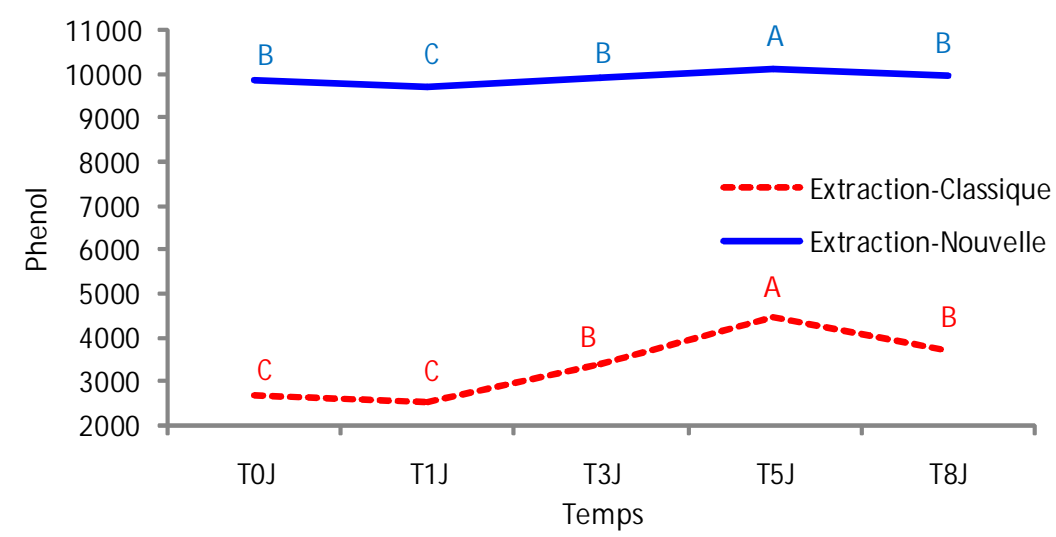

Figure 1: Evolution des teneurs globales en phénols des racines après inoculation du F.O.E. en fonction des techniques d'extraction 
Tableau 2: Comparaison en fonction de la technique d'extraction, des teneurs phénoliques globales des racines de clones tolérants et de clones sensibles non inoculés: Moyenne mesurée sur cinq individus

\begin{tabular}{|c|c|c|c|c|c|c|}
\hline & Source de variation & ddl & $\begin{array}{c}\text { Somme } \\
\text { des carrés }\end{array}$ & $\begin{array}{c}\text { Carré } \\
\text { moyen }\end{array}$ & $\begin{array}{c}\text { F de } \\
\text { Fisher }\end{array}$ & $\operatorname{Pr}>\mathrm{F}$ \\
\hline \multirow{8}{*}{ Couple A } & Clone & 1 & 4360818,517 & 4360818,517 & 517,958 & $<0,0001$ \\
\hline & Extraction & 1 & 84647039,905 & 84647039,905 & 10053,992 & $<0,0001$ \\
\hline & \multirow{6}{*}{ Test de Newman-Keuls (SNK) } & & Modalités & Moyenne & \multicolumn{2}{|c|}{ Regroupements } \\
\hline & & & Nouvelle & 9177,500 & \multirow[t]{2}{*}{$A$} & \\
\hline & & & Classique & 5053,700 & & B \\
\hline & & & Modalités & Moyenne & \multicolumn{2}{|c|}{ Regroupements } \\
\hline & & & LMC 22 & 7407,000 & \multirow[t]{2}{*}{ A } & \\
\hline & & & LMC 077 & 6877,182 & & B \\
\hline \multirow{9}{*}{ Couple B } & Source de variation & ddl & $\begin{array}{l}\text { Somme } \\
\text { des carrés }\end{array}$ & $\begin{array}{c}\text { Carré } \\
\text { moyen }\end{array}$ & $\begin{array}{l}\text { F de } \\
\text { Fisher }\end{array}$ & $\mathrm{Pr}>\mathrm{F}$ \\
\hline & Clone & 1 & 3380,000 & 3380,000 & 1352,000 & $<0,0001$ \\
\hline & Extraction & 1 & 146232320,000 & 146232320,000 & 58492928,000 & $<0,0001$ \\
\hline & \multirow{6}{*}{ Test de Newman-Keuls (SNK) } & & Modalités & Moyenne & \multicolumn{2}{|c|}{ Regroupements } \\
\hline & & & Nouvelle & 9317,000 & \multirow[t]{2}{*}{ A } & \\
\hline & & & Classique & 3909,000 & & B \\
\hline & & & Modalités & Moyenne & \multicolumn{2}{|c|}{ Regroupements } \\
\hline & & & LMC 119 & 6626,000 & \multirow[t]{2}{*}{ A } & \\
\hline & & & LMC 165 & 6600,000 & & $\mathrm{~B}$ \\
\hline \multirow{9}{*}{ Couple C } & Source de variation & ddl & $\begin{array}{c}\text { Somme } \\
\text { des carrés }\end{array}$ & $\begin{array}{c}\text { Carré } \\
\text { moyen }\end{array}$ & $\begin{array}{l}\text { F de } \\
\text { Fisher }\end{array}$ & $\mathrm{Pr}>\mathrm{F}$ \\
\hline & Clone & 1 & 416161,250 & 416161,250 & 166464,500 & $<0,0001$ \\
\hline & Extraction & 1 & 184923211,250 & 184923211,250 & 73969284,500 & $<0,0001$ \\
\hline & \multirow{6}{*}{ Test de Newman-Keuls (SNK) } & & Modalités & Moyenne & \multicolumn{2}{|c|}{ Regroupements } \\
\hline & & & Nouvelle & 9881,500 & \multirow[t]{2}{*}{$\mathrm{A}$} & \\
\hline & & & Classique & 3800,000 & & B \\
\hline & & & Modalités & Moyenne & \multicolumn{2}{|c|}{ Regroupements } \\
\hline & & & LMC 062 & 6985,000 & \multirow[t]{2}{*}{ A } & \\
\hline & & & LMC 204 & 6696,500 & & B \\
\hline
\end{tabular}

Tableau 3: Comparaison en fonction de la technique d'extraction, des teneurs phénoliques globales des racines de clones tolérants et de clones sensibles inoculés : Moyenne mesurée sur cinq individus

\begin{tabular}{|c|c|c|c|c|c|}
\hline Source & ddl & $\begin{array}{c}\text { Somme des } \\
\text { carrés }\end{array}$ & $\begin{array}{c}\text { Carré } \\
\text { moyen }\end{array}$ & F de Fisher & $\operatorname{Pr}>\mathrm{F}$ \\
\hline Clone & 1 & 16000000,000 & 16000000,000 & 47,727 & $<0,0001$ \\
\hline Extraction & 1 & 1072562500,000 & 1072562500,000 & 3199,417 & $<0,0001$ \\
\hline Temps & 4 & 16546250,000 & 4136562,500 & 12,339 & $<0,0001$ \\
\hline \multirow{6}{*}{$\begin{array}{l}\text { Test de } \\
\text { Newman-Keuls } \\
\text { (SNK) }\end{array}$} & Modalités & Moyenne & \multicolumn{3}{|c|}{ Regroupements } \\
\hline & LMC 022 & 7035,000 & \multirow{2}{*}{\multicolumn{2}{|c|}{ A }} & \\
\hline & LMC 074 & 6235,000 & & & $B$ \\
\hline & Modalités & Moyenne & \multicolumn{3}{|c|}{ Regroupements } \\
\hline & Nouvelle & 9910,000 & \multirow{2}{*}{\multicolumn{2}{|c|}{$\bar{A}$}} & \\
\hline & Classique & 3360,000 & & & $\mathrm{~B}$ \\
\hline
\end{tabular}

\subsection{Comparaison de la fongitoxicité des phénols pour le marquage de la tolérance des clones}

L'analyse de la fongitoxicité des phénols des plantules inoculées extraites par les deux méthodes a montré d'une part l'existence de différences significatives entre les niveaux de fongitoxicité des composés phénoliques en fonction des techniques d'extraction. La technique nouvelle a permis de révéler des niveaux de fongitoxicité bien plus élevés que les niveaux de fongitoxicité qui ont été révélés par la technique classique. D'autre part, les niveaux de fongitoxicité des composés phénoliques du clone tolérant LMC 022 inoculé ont été significativement plus élevés 
que les niveaux de fongitoxicité du clone sensible LMC 074 inoculé (Tableau 4). Des variations significatives de niveaux de fongitoxicité des composés phénoliques ont été observées au cours du temps toujours avec des valeurs plus élevées avec la méthode d'extraction nouvelle contrairement à la méthode d'extraction classique (Figure 2).

Tableau 4: Comparaison en fonction de la technique d'extraction, des fongitoxicité globales des racines de clones tolérants et de clones sensibles inoculés : Moyenne mesurée sur cinq individus

\begin{tabular}{lccccc}
\hline Source & ddl & Somme des carrés & Carré moyen & F de Fisher & Pr $>$ F \\
\hline Clone & 1 & 3025,000 & 3025,000 & 383,799 & $<0,0001$ \\
Extraction & 1 & 400,000 & 400,000 & 50,750 & $<0,0001$ \\
Temps & 4 & 184,000 & 46,000 & 5,836 & 0,000 \\
\hline \multirow{3}{*}{ Test de } & Modalités & Moyenne & Regroupements & Pr $>$ F \\
\cline { 2 - 6 } Newman-Keuls & Nouvelle & 34,600 & $\mathrm{~A}$ & & \\
(SNK) & Classique & 30,600 & & $\mathrm{~B}$ & \\
\cline { 2 - 6 } & Modalités & Moyenne & Regroupements & \\
\cline { 2 - 6 } & LMC 022 & 38,100 & $\mathrm{~A}$ & $\mathrm{~B}$ & \\
\hline
\end{tabular}

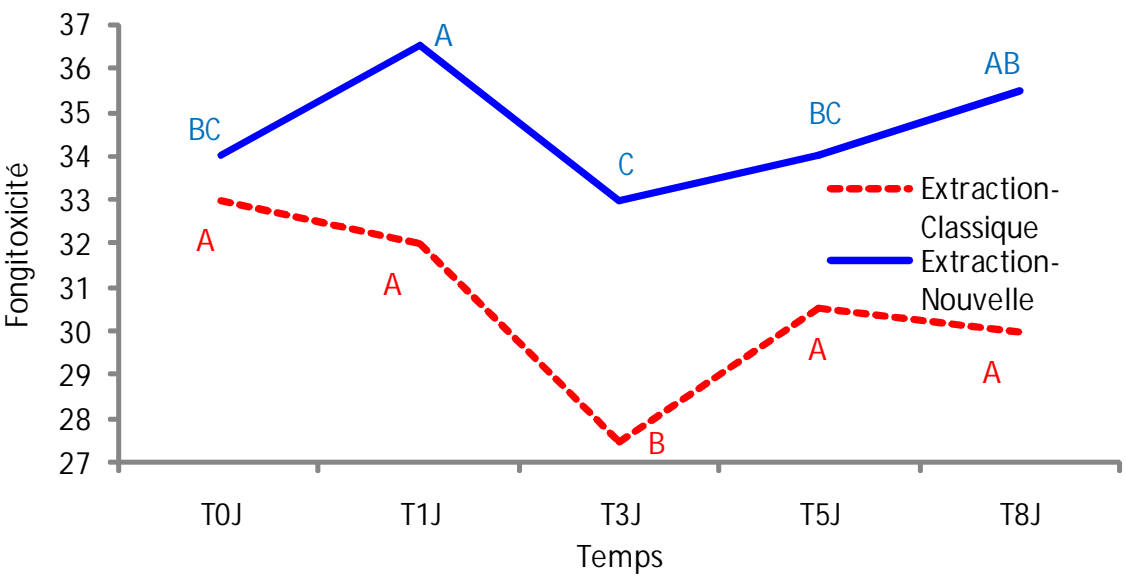

Figure 2: Evolution des teneurs globales en phénols des racines après inoculation du F.O.E. en fonction des techniques d'extraction

\section{Discussion}

Plus le taux de phénol étudié sera grand plus la probabilité de mettre en évidence de bons marqueurs phénoliques de la résistance à la fusariose sera forte. Dans ce but, le premier facteur considéré a été l'amélioration de l'extraction. Celleci dépend du solvant utilisé. Les résultats ont montré que la technique d'extraction la plus efficace consistait en une macération de $30 \mathrm{mn}$ à ébullition sous reflux, dans un mélange Ethanol $70 \%$ - Eau $30 \%$, en présence d'un antioxydant, le métabisulfite de sodium (MBS) qui a été ajouté au solvant de macération afin d'éviter les phénomènes d'oxydation des phénols au cours de leur extraction. Cette méthode a permis en effet d'extraire en moyenne plus d'une fois de phénols que la macération classique dans l'éthanol pur. Elle a permis d'accéder aux phénols solubles en solution dans le milieu cellulaire étudié jusqu'à présent (Taquet, 1985 ; Diabaté, 1985 ; 1990) et aussi aux phénols liées à la paroi cellulaire dont le rôle dans le processus de résistance aux maladies a été reconnu (Ampomah \& Fried, 1988).

L'analyse des résultats a montré que la réaction de défense du palmier à huile contre la fusariose vasculaire se manifestait par la synthèse de 
composés phénoliques. En outre, l'évolution des teneurs phénoliques a indiqué des quantités plus importantes de phénols chez les clones tolérants que chez les clones sensibles. L'analyse des résultats a aussi indiqué une activité antifongique plus marquée chez les clones tolérants que chez les clones sensibles. Ces résultats corroborent ceux de Renard et al., (1986) qui ont constaté dans les racines de palmiers inoculés, un accroissement significatif de dérivés de l'acide benzoïque. Ces substances ont inhibé in vitro la croissance du F.O.E.

En ce qui concerne l'anabolisme phénolique, une accumulation importante de phénols en réponse à une infection de la plante résistante a été généralement constatée. Ce phénomène peut concerner aussi bien les phénols simples que les polymères phénoliques (Ledeme, 1991, Zhu \& Yao, 2004). Chez les plantes sensibles par contre, l'anabolisme phénolique suivant l'infection ne s'accompagne d'aucun accroissement de l'accumulation phénolique dans les tissus racinaires de l'hôte. Qualitativement, les extraits alcooliques réalisés à partir de ces tissus s'avèrent sans effet sur la croissance in vitro du champignon (Bell et al., 1984 ; Basham, 1986 ; Trojaak \& Berbec, 2005). Les résultats que nous avons obtenus chez le palmier à huile confirment ces hypothèses et augure une possibilité de mise en place d'un test précoce à la fusariose du palmier à huile.

\section{Conclusion}

La ré-investigation des méthodes d'extraction et de dosages des composés phénoliques a permis de dégager les acquis suivants:

- d'abord une méthode de broyage à l'azote liquide $\left(-160^{\circ} \mathrm{C}\right)$ qui a permis d'obtenir une poudre fine et homogène à partir de $5 \mathrm{~g}$ de racines. De plus la température de l'azote liquide a permis de maintenir les tissus racinaires congelés durant tout le temps du broyage, ce qui a limité les risques de dégradation des produits phénoliques.

- ensuite l'addition du Métabisulfite de Sodium à $2 \%$ dans le solvant de macération a empêché l'oxydation des phénols au cours de leurs extractions.

- en outre, l'extraction des racines broyées dans un mélange Ethanol-Eau (70 \%-30
$\%, v / v)$ a donné après dosage un extrait moins riche en huile, et plus riche en phénols. Ce qui a entraîné une activité antifongique plus grande contrairement à la méthode d'extraction classique avec l'Ethanol pur. Ainsi la discrimination entre clones tolérants et clones sensibles a été facilitée.

- le dosage des phénols a aussi montré qu'il existait une réponse phénolique des plantules à l'inoculation du Fusarium oxysporum f $\mathrm{sp}$ elaeidis les jours qui suivaient cette inoculation. Cette réponse était plus importante avec la nouvelle méthode d'extraction que la méthode classique.

Enfin, ces résultats sont particulièrement importants dans l'optique de la mise au point d'un test précoce détection de la tolérance à la fusariose à partir du dosage des phénols racinaires.

\section{Références citées}

Allou K., Ahoussou N., Ake S., Diabate S. \& De Franqueville H., 2003. Comportement de clones de palmier à huile au champ en zones de haute densité de F.O.E en Côte d'Ivoire. Agron. Afr. 15 (1) : 29-38.

Ampomah YAW A. \& John FRIED., 1988. Insoluble phenolic compounds and resistance of patato tuber disc to phytophthora and phoma. Phytochemistry, 27, 8: 2533-2541.

Basham Y., 1986. Phenols in cotton seedlings resistance and susceptible to Alternaria macrospora. J. Phytopathol 116 : 1-10.

Bastide P., 1988. Influence in vitro des composes phénoliques des jeunes feuilles du pécher. Prunus persica (1) Batsch, sur le puceron vert du pecher, Myzus persicae sulzer. Agronomie 8 (9) : 787-792.

Bell J.N., Dixon R.A., Bailey J.A., Rowell P.M. \& Lamb C.J., 1984. Differential induction of chalcone synthase mRNA activity at the onset of phytoalexin accumulation in compatible and incompatible plant pathogen interactions. Proc. Natl. Acad. Sci 81 : 3384-3388.

Diabate S., 1985. Etude comparative des réactions de défense contre la fusariose vasculaire. DEA 
de phytopathologie - Paris 11 et Paris 6. INA PARIS - GRIGNON, France. 10 pp.

Diabate S., Taquet B., Renard J.L., De Franqueville H. \& Reiser P., 1990. Analyse en CLHP des substances produites par le palmier à huile au cours de l'infection par le Fusarium oxysporum f. sp. elaeidis. Perspective pour la sélection. Oléagineux 45 (2) : 49-53.

Diabate S., Ledeme P., De Franqueville H. \& Kouame B., 1992. Effet d'une souche avirulente du Fusarium oxysporum f sp elaeidis .E sur l'expression de la fusariose et sur les propriétés fongitoxiques des extraits racinaires de palmier à huile. In : Interactions plantes microorganismes. 17-27 février 1992. DAKAR (SENEGAL) : Fondation internationale pour la science, pp. 307-320.

Durand-Gasselin T., Kouame K. R., Cochard B., Adon B. \& Amblard P., 2000. Diffusion variétale du palmier à huile (Elaeis guineensis Jacq). Oléagineux Corps gras Lipides 7 (2) : 207-214.

Franqueville H. (De). \& Diabate S., 2004. Notes on oil palm vascular wilt. Communication présentée au Malaysian Palm oil Board (MPOB) du 18 au 19 Mai 2004 Kuala Lumpur (Malaisie).

Ledeme P., 1991. Métabolisme phénolique de la fleur d'œillet (Dianthus caryophyllus L.).
Relation avec la création et le diagnostic des variétés. Thèse doctorat université Claude Bernard, Lyon France, 240 pp.

Marigo G., 1973. Sur une méthode de fractionnement et d'estimation des composés phénoliques chez les végétaux. Analusis, $2: 10-110$

Renard J.L. \& Andre R., 1986. La fusariose du palmier à huile. Phytoma Défense des cultures. 44-46.

Taquet B., 1985. Les mécanismes physiologiques de la réaction de défense du palmier à huile contre la fusariose vasculaire. Application à la recherche de nouveaux moyens de lutte. Thèse de Doctorat 3è cycle. Université Paris 6, France. 151 pp.

Trojak G. A. \& Berbec A., 2005. Potential of Nicotiana glauca (Grah) as a source of resistance to black root. Thielaviopsis basicola (Berk and Broome). Ferr. In : Tobacco improvement. Plant Breed. 124 : 507-510.

Zhu H. M. \& Yao Q., 2004. Localized and systemic increase of phenols in tomato roots induced by Glomus versiforme inhibits Ralotronia solanacearum. J. Phytopathol. 152 : 537-542. 